\title{
A Pedigree Report of a Rare Case of Weill- Marchesani Syndrome with New Compound Heterozygous LTBP2 Mutations
}

\section{ZhiHong Lin* \\ MinJuan Zhu* \\ HongWei Deng}

Department of Strabismus \& Pediatric Ophthalmology, Shenzhen Eye Hospital Affiliated to Jinan University, The School of Optometry of Shenzhen University, Shenzhen, 518000, Guangdong Province, People's Republic of China

*These authors contributed equally to this work
Correspondence: HongWei Deng Department of Strabismus \& Pediatric Ophthalmology, Shenzhen Eye Hospital Affiliated to Jinan University, The School of Optometry of Shenzhen University, No. 18 of Zetian Street, Futian District, Shenzhen, 518000, People's Republic of China

Tel +86075523959542

Fax +86075523959500

Email denghwdr@2Icn.com
Background: Weill-Marchesani syndrome (WMS) is an autosomal inherited connective tissue disease. Clinical manifestations include microspherophakia (MSP), high myopia, ectopia lentis, open-angle glaucoma, short stature, short fingers, joint stiffness, and (occasionally) cardiovascular defects. At present, a total of four pathogenic gene loci related to WMS have been found: ADAMTS10, ADAMTS17, FBN1, and LTBP2.

Case Report: The patient was a five-year-old girl whose eyesight had become progressively worse for three years before her parents brought her to the hospital. Computer optometry showed high myopia in both eyes, while a slit lamp examination found that the anterior chamber of both eyes was shallow, and the lens was in a state of dislocation (ectopia lentis). An IOLMaster examination revealed that the lens was spherical (MSP), and the lens thickness (LT) was $5.36 \mathrm{~mm}$. Corneal topography showed that the angle kappa was $0.18 \mathrm{~mm}$ in the right eye (OD) and $0.30 \mathrm{~mm}$ in the left eye (OS). An intraocular pressure (IOP) (OD: $26.5 \mathrm{mmHg}$, OS: $30.6 \mathrm{mmHg}$ ) examination showed that the fundus cup to disc ratio was normal, but secondary glaucoma caused by lens dislocation could be considered. The IOP was maintained within a normal range using antihypertensive drugs. The patient's younger sister also had a dislocation of MSP. Gene detection showed a heterozygous mutation in the LTBP2 gene [c.3672delC:p.Thr1225fs and c.3542delT:p.Met1181fs], and a diagnosis of WMS-like syndrome was confirmed.

Conclusion: WMS syndrome is rare, and the mutation of the LTBP2 gene has not been previously recorded in the GnomAD (Genome Aggregation Database) of East Asia. This case report provides some reference for studying the mechanism of WMS and WMS-like syndrome caused by an LTBP2 gene mutation.

Keywords: Weill-Marchesani syndrome, microspherophakia, ectopia lentis, LTBP2, compound heterozygous, secondary glaucoma

\section{Background}

Weill-Marchesani syndrome (WMS) is an autosomal inherited connective tissue disease. It can be either autosomal recessive (AR) or autosomal dominant (AD), depending on the genetic pattern. ${ }^{1}$ The prevalence of WMS in different populations is $1: 100,000 .^{2}$ Clinical manifestations include microspherophakia (MSP), high myopia, ectopia lentis (EL), open-angle glaucoma, short stature, short fingers, joint stiffness, and (occasionally) cardiovascular defects.

When the above clinical symptoms occur, WMS's presence is likely, and relevant genetic testing can be conducted for confirmation. At present, a total of 
four pathogenic gene loci related to WMS have been found: ADAMTS10, ADAMTS17, LTBP2, and FBN1. The first three are connected with AR inheritance, which accounts for $45 \%$ of WMS cases, while the last is associated with dominant inheritance, which accounts for $39 \%$ of WMS cases; the remaining $16 \%$ are sporadic cases. Although the genetic pattern and the pathogenic gene may be different, their clinical manifestations are the same.

\section{Case Report}

The child's parents had observed the child's poor eyesight for three years, so they brought the child to Shenzhen Eye Hospital for medical treatment. The child's visual acuity was examined using computer optometry, and the following results were obtained: OD: $-19.00 \mathrm{DS}$ (spherical lens) -1.50 DC (cylindrical lens) $\times 15$; OS: -19.50 (spherical lens) -3.00 (cylindrical lens) $\times 165$. The slit-lamp examination showed that both eyes' anterior chambers were shallow, the anterior chamber depth was about 2 CT. CT is the corneal thickness seen under a slit lamp, 1 $\mathrm{CT}=1$ corneal thickness, which is a rough method to estimate the depth of the anterior chamber.

The sclera, conjunctiva, and cornea were normal, but the child's lens was EL. After mydriasis, the anterior segment photograph showed that the EL was tremulous and in a state of dislocation under the nasal septum, and the equatorial part of the lens could be seen in the pupil area (Figure 1). An IOLMaster examination found that the lens was spherical, and the LT was $5.36 \mathrm{~mm}$ (Figure 1). A corneal topography examination showed that the right eye angle kappa was $0.18 \mathrm{~mm}$, whereas the left eye angle kappa was $0.30 \mathrm{~mm}$. Scanning laser ophthalmoscope (SLO) examination showed that the fundus retina was generally normal (Figure 1). SLO examination is performed using the red and green laser irradiation on the retina and then capturing its reflected
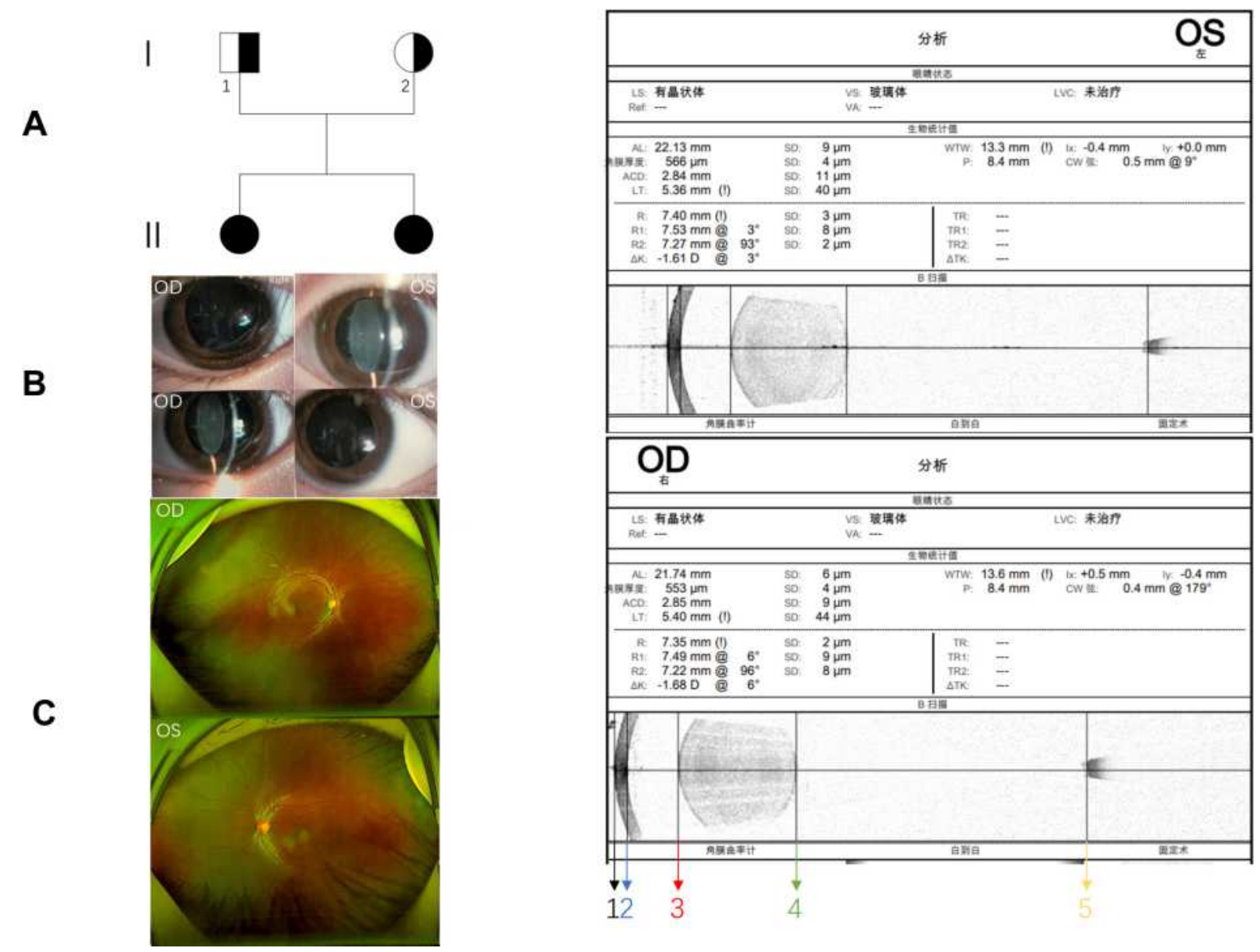

Figure I A family with Weill-Marchesani syndrome: (A) pedigree of the family with Weill-Marchesani syndrome; (B) the anterior segment photograph shows the dislocation of the microspherophakia (MSP) to the lower part of the nose; (C) SLO (Panoramic Ophthalmoscope-Daytona P200T) showing that the fundus of the patient was normal, and there was no obvious fundus retinal change; (D) IOLMaster (ZEISS IOLMaster 700) showing that the patient had MSP with normal AL (axial length). In the picture, black line I to yellow line 5 is the AL (axial length), red line 3 to green line 4 is the LT (lens thickness), blue line 2 to red line 3 is the ACD (anterior chamber depth), black line I to blue line 2 is the corneal thickness. 
light to obtain a wide-angle picture of the fundus. The first IOP measured by a non-contact tonometer was normal. The non-contact intraocular pressure meter uses high-speed gas to spray on the cornea's surface to measure the intraocular pressure through the change of the shape of the cornea.

As the child would not cooperate with subsequent examinations, she was given glasses to correct her vision, surgery was postponed, and she was diagnosed as "EL OU (both eyes), spherical lens OU, ametropia OU." One year later, when the child came back to the hospital for re-examination on October 22, 2020, it was found that the intraocular pressure was elevated, and the fundus cup to disc ratio was normal (OD: $26.5 \mathrm{mmHg}$, OS: $30.6 \mathrm{mmHg}$ ). The fundus cup to disc ratio refers to a physiological depression in the central part of the optic nerve papilla. It has a certain reference value in the diagnosis of glaucoma. Thus, secondary glaucoma caused by lens dislocation was considered. The IOP was controlled by antihypertensive drugs, Carteolol Hydrochloride eye drops, and fell within the normal range. The patient's sister was also found to have poor eyesight, and after being seen by a doctor, she was diagnosed as having MSP.

After obtaining the consent of the family members and the approval of the Ethics Committee of Shenzhen Eye Hospital, we extracted blood from the patient, her sister,

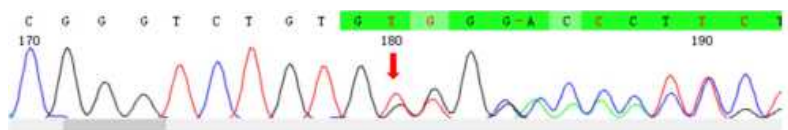

(A) Patient (c.3672delC:p.T hr 1225fs)

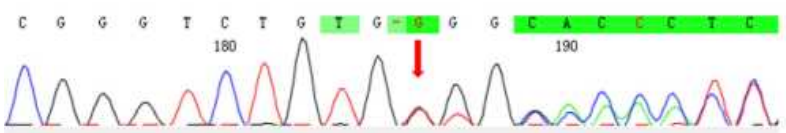

(A) Sister (c.3672delC:p.T hr 1225fs)

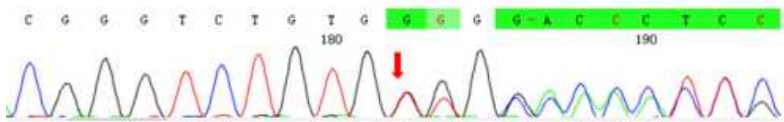

(A) Father (c.3672delC:p.T hr 1225fs)

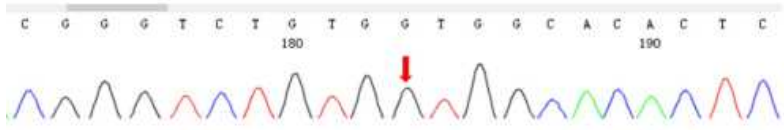

(A) Mother (c.3672) and their parents. Second-generation sequencing of the genes was then performed. High-throughput sequencing and analysis were performed using the patient's clinical characteristics (low-quality mutations were filtered during data analysis to ensure accurate analysis), and two heterozygous mutations were found in the latent transforming growth factor beta-binding protein 2 (LTBP2) gene on chromosome 14 of the patient and her sister (Figure 2). Variant 1 is a frameshift deletion variant c.3672delC:p.Thr1225fs, while Variant 2 is a frameshift deletion variant c.3542delT:p.Met1181fs. WMS is an AR genetic disease, and we identified a possible pathogenic Variant 1 at the trans-position of this variant.

The verification results of the first-generation sequencing showed that Variant 1 was inherited from the father, and Variant 2 was inherited from the mother, which constituted a complex heterozygous mutation. Neither of these two variants has been previously reported in the literature.

\section{Discussion}

MSP is a rare ocular clinical manifestation. Enlargement or dislocation of MSP may lead to pupil obstruction and secondary angle-closure glaucoma. The occurrence of MSP is related to the abnormal development of the mesoderm in the embryonic stage. The lens is spherical at five

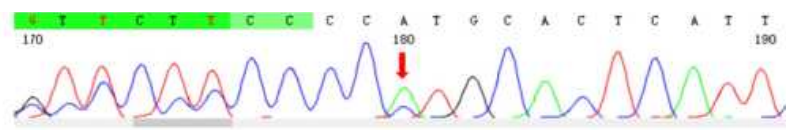

(B) Patient ( c.3542delT:p.M et $1181 \mathrm{fs}$ )

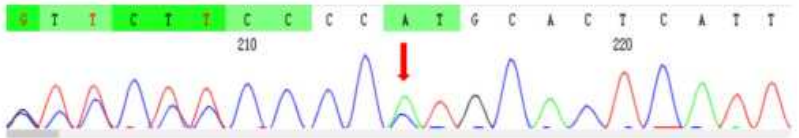

(B) Sister (c.3542delT:p.M et $1181 \mathrm{fs}$ )

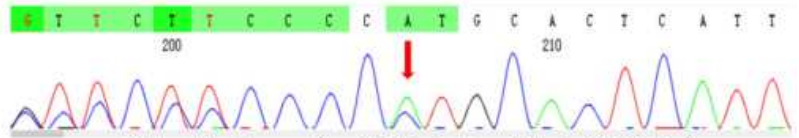

(B) Mother ( c.3542delT:p.M et $1181 \mathrm{fs}$ )

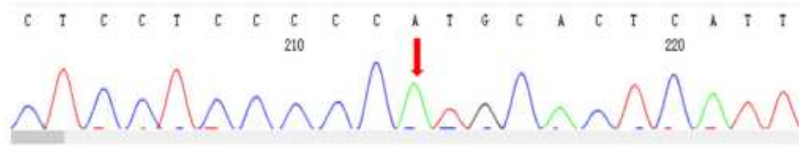

(B) Father (c.3542)

Figure 2 Mutations in the LTBP2 gene of the patient and her family. The waveforms of the four colors represent the signal strength of the four bases, A is green, T is red, $\mathrm{G}$ is black, and $\mathrm{C}$ is blue. Under normal circumstances, the peak is clear, and the distance between the two peaks is uniform. (A and B) Variant $\mathrm{I}$ and Variant 2 constitute a compound heterozygous missense mutation: (A) the mother had no heterozygous frameshift deletion, the red arrow was the normal peak of $G$, while the patient, her sister, and father had the heterozygous frameshift deletion, so their $G$ peaks became shorter and the $T$ peaks mixed together at the red arrows. (B) The father had no heterozygous frameshift deletion. The red arrow was the normal peak of $A$, while the patient, her sister, and mother found the heterozygous frameshift deletion, so their A peaks became shorter and the $C$ peaks mixed together at the red arrows. 
to six months of embryonic development of the eye and then gradually develops into a biconvex shape due to the development of the ciliary body and suspensory ligament of the mesoderm. ${ }^{3}$ In MSP, the spherical shape is maintained due to the abnormal development of the mesoderm and suspensory ligament. MSP often occurs in WMS, Marfan syndrome, Alport syndrome, and oculodentodigital syndrome, but a few cases have been reported as an isolated defect. Among these rare diseases, WMS and Marfan syndrome are the most common.

Although both of them have ocular manifestations of MSP, Marfan syndrome is also characterized by being unbalanced, having slender, long limbs, fingers, and toes due to the excessive extension of the connective tissue, and those suffering from this syndrome tend to be taller than average, which is opposite of people with WSM. ${ }^{4}$ It is necessary to carry out molecular genetic testing of the corresponding genes to make a final MSP diagnosis.

LTBP2 primarily affects the production of the extracellular matrix (ECM) ${ }^{5}$ It is expressed in elastic tissue and binds to fibrin 1, which contains microfibrils. ${ }^{6}$ Microfibers exist not only in elastic tissues, such as ligaments, valves, and lens capsules but also in tissues lacking elastin, such as the ciliary zone. Microfilaments are relatively rigid elastic films and can anchor at the interface between

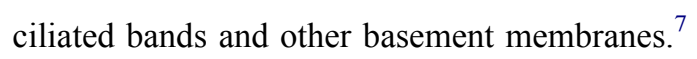

Variant 1 is the deletion variation c.3672delC:p. Thr1225fs. This variation causes the LTBP2 gene to be shifted from the 1,225th codon and produces a termination codon in advance. Variant 2 is the deletion variation c.3542delT:p.Met1181fs, which causes the code shift of the LTBP2 gene from the 1,181st codon and produces a termination codon in advance. Variants 1 and 2 constitute a compound heterozygous missense mutation, which will lead to a partial loss of function of the LTBP2 gene, causing the deterioration of the microfilament stability of the suspensory ligament and the lens capsule and, finally, resulting in a series of ocular clinical WMS symptoms, such as MSP, EL, and secondary pupil block glaucoma. Both the father and mother of the case in question have normal phenotypes. This means that neither c.3672delC:p. Thr1225fs (the father) nor c.3542delT:p.Met1181fs (the mother) can cause the disease phenotype alone.

Identifying these pathogenic gene mutation sites will help complement the genetic diagnosis of WMS and provide a new direction for prenatal diagnosis to prevent more congenital eye diseases.

\section{Ethics Approval and Consent to Participate}

This study was conducted in accordance with the declaration of Helsinki.This study was conducted with approval from the Ethics Committee of Shenzhen Eye Hospital affiliated to Jinan University.A written informed consent was obtained from legal guardians of all participants.

\section{Consent for Publication}

Consent for publication was obtained from legal guardians of all participants whose data are included in this manuscript.

\section{Funding}

International Science and Technology Cooperation Research Project of Shenzhen Science and Technology Innovation Committee (GJHZ20190821113401670); Sanming Project of Medicine in Shenzhen (SZSM201812090); Supported by Shenzhen Fund for Guangdong Provincial High-level Clinical Key Specialties (No. SZGSP014).

\section{Disclosure}

All authors declare that the work is original and has not been submitted or published elsewhere. None of the authors have any financial disclosure or conflicts of interest.

\section{References}

1. Faivre L, Dollfus H, Lyonnet S, et al. Clinical homogeneity and genetic heterogeneity in Weill-Marchesani syndrome. Am J Med Genet A. 2003;123A(2):204-207. doi:10.1002/ajmg.a.20289

2. Marzin P, Cormier-Daire V, Tsilou E. Weill-Marchesani syndrome. In: Adam MP, Ardinger HH, Pagon RA, et al., editors. Genereviews $\left({ }^{\circledR}\right)$. Seattle, WA: University of Washington, Seattle Copyright (C) 1993-2020, University of Washington, Seattle. GeneReviews is a registered trademark of the University of Washington, Seattle. All rights reserved; 1993.

3. Chan RT, Collin HB. Microspherophakia. Clin Exp Optom. 2002;85 (5):294-299. doi:10.1111/j.1444-0938.2002.tb03085.x

4. Dureau P. Pathophysiology of zonular diseases. Curr Opin Ophthalmol. 2008;19(1):27-30. doi:10.1097/ICU.0b013e3282f29f01

5. Faivre L, Gorlin RJ, Wirtz MK, et al. In frame fibrillin-1 gene deletion in autosomal dominant Weill-Marchesani syndrome. J Med Genet. 2003;40(1):34-36. doi:10.1136/jmg.40.1.34

6. Karoulias SZ, Beyens A, Balic Z, et al. A novel ADAMTS17 variant that causes Weill-Marchesani syndrome 4 alters fibrillin-1 and collagen type I deposition in the extracellular matrix. Matrix Biol. 2020;88:1-18. doi:10.1016/j.matbio.2019.11.001

7. Haji-Seyed-Javadi R, Jelodari-Mamaghani S, Paylakhi SH, et al. LTBP2 mutations cause Weill-Marchesani and Weill-Marchesani-like syndrome and affect disruptions in the extracellular matrix. Hum Mutat. 2012;33(8):1182-1187. doi:10.1002/humu.22105 


\section{Publish your work in this journal}

Risk Management and Healthcare Policy is an international, peerreviewed, open access journal focusing on all aspects of public health, policy, and preventative measures to promote good health and improve morbidity and mortality in the population. The journal welcomes submitted papers covering original research, basic science, clinical \& epidemiological studies, reviews and evaluations, guidelines, expert opinion and commentary, case reports and extended reports. The manuscript management system is completely online and includes a very quick and fair peer-review system, which is all easy to use. Visit http://www.dovepress.com/testimonials.php to read real quotes from published authors.

Submit your manuscript here: https://www.dovepress.com/risk-management-and-healthcare-policy-journal 